\title{
Vereins- und Verbandsregister
}

1. Österreichische Damenfussball-Union 91, 258

1. Wiener Damenfußballklub Kolossal 91

Achduth 76

Achim 76

SC Admira 1, 18, 40, 107-110, 113-117, 119, $130,131,134,243,247,252,324,326$

Aero-Club 1

SK Ahawath Zion 82

Akademischer Sportklub 239

Allgemeiner österreichischer Skiverband 34, 221

Allgemeiner Turn- und Sportverein; siehe Deutsch-österreichischer Turnverein

Arbeiterbund für Sport und Körperkultur in Österreich (ASKÖ) 79, 92, 93, 191, 192, 218, 237, 257, 276, 317

Ari 76

FC Arsenal London 255

FK Austria 14, 20-22, 40, 93, 98-100, 104, $106,107,117-119,122,123,125,126$, 131, 132, 144, 179, 202, 237, 246, 247, $249,252,254,256,263,298-315,327$

Austria Klagenfurt 38

Bar Kochba 77, 82

Bar Kochba Berlin 87

Bar Kochba Frankfurt 87

Bergfreunde; siehe Naturfreunde

SC Bernhard Altmann 4, 83, 320, 321

SK Blau-Weiß Hamburg 87

Brigittenauer AC (BAC) 98, 99, 254

Bund Deutscher Radfahrer Oesterreichs

(B.D.R.Oes.) 27-29

Christlich-deutsche Turnerschaft $67,69,86$, 277

Comité zur Beschickung der Pariser Olympischen Spiele 72

Damenschwimmclub Austria 236

Deutsche Jungmannschaft Währing 41

Deutscher Makkabi-Kreis 87, 228, 284, 285

Deutscher Radfahr-Bund (D.R.B.) 27
Deutscher Reichsbund für Leibesübungen

(DRL) 278-280, 287

Deutscher Sportklub Villach 38

Deutscher Sportverein; siehe Wiener SportClub

Deutscher Sportverrein Leoben (DSL) 38, 42, 45,84

Deutscher Turnerbund 26, 27, 67, 69, 85, 248, 273, 276, 279

Deutscher und Oesterreichischischer Alpenverein (DuOeAV) 17, 23, 24, 30-32, 44, 67, 70, 190, 196, 197, 207, 221, 280

- Sektion Austria 32

- Sektion Donauland 32, 281, 282

Deutsch-österreichischer Turnverein 12, 67, $69,71,73$

DFC Prag 116

Donawitzer SC 43

Eishockeyklub Engelmann (E.K.E.) 321

Eishockeyverband 69, 253

Erster Badener Jüdischer Sportclub Unitas 84,87

Erster Simmeringer Sportclub (ISSC) 93, 107, 123, 124, 252

Erster Wiener Amateur-Schwimm-Club (EWASC) 35-37, 45, 142, 292

Erster Wiener Jüdischer Turnverein 26, 71, 73

FC Wien 18, 93

Fédération Internationale de Football Association (FIFA) 252, 255, 259, 327

Fédération Sportive Féminine Internationale 257

First Vienna Football Club 21, 22, 40, 65, 71, $95,99,100,104,107,117,144-157,180$, 189, 218, 221, 222

Floriana FC (Malta) 111

Floridsdorfer Athletiksport-Club (FAC) 1, 40, 107-114, 128, 130, 131, 135, 141, 212, $318,319,326,329$

Grasshopper Club Zürich (Grasshoppers) 256

Grazer Athletiksport-Klub (GAK) 38

○ Open Access. (๑) 2019 Bernhard Hachleitner, Matthias Marschik und Georg Spitaler, publiziert von De Gruyter. (@) BY Dieses Werk ist lizenziert unter der Creative Commons Attribution 4.0 International Lizenz (CC BY 4.0). https://doi.org/10.1515/9783110553314-014 
Hagibor 76, 77, 82, 84

Hagibor Prag 249

Hagibor-Makkabi; siehe Hagibor

SC Hakoah 2, 4, 12, 13, 16, 17, 20-22, 30, $35,37,42,66,70,71,74-81,83,85-$ $88,93,95-99,103,106,107,109,117$, $119,125,128-133,135,153,154,185$, 186, 196, 206, 214, 218, 231, 237-239, 242, 245-247, 249, 250, 263, 264, 277, 282, 284, 285, 288-298, 300, 312, 318, 321-323, 328, 330

- Fußballklub (FC) Hakoah 87, 96, 128, 237, 289, 290, 293, 296

- Schwimmklub Hakoah 70, 87, 88, 90, 96, 231, 277, 281, 283, 289, 290, 295, 296

- Touristik \& Skiklub Hakoah 87, 281, 289, 293, 294, 296, 297

Hakoah Eisenstadt 84

SC Hakoah Graz 13, 38, 42, 73, 77, 79, 81, 84,87

Hakoah Innsbruck 84, 87, 283

Hakoah Krems 84

Hakoah Leoben 84

Hakoah Linz 13

Handballbund 69

Hanizachon 77

Hapoel 16, 77, 83, 87, 277

Hapoel Hechadasch 87, 277

SC Hasmonea 16, 76, 78, 79, 81-85, 96, 98, 99, 237, 244

Hauptverband für Körpersport 34, 35, 70, 150, 220, 230, 254, 276

Hazair-Makkabäa 77, 80, 87, 218, 282

Hechawer 76, 78-81

ASV Hertha 81

Hockeyclub Blau Weiß 77

Internationaler Skiverband (FIS) 34

Internationales Olympisches Comité (IOC) 35, 150, 244, 254

Italienischer Fußballverband 259, 327

JSV Makkabi 81, 85, 96, 99

Jüdischer Arbeitersportklub Borochow 77, 79 Jüdischer Arbeiter-Sportklub Cheruth 82

Jüdischer Athletik Club (J.A.C.) 77, 78, 82 Jüdischer FC 96
Jüdischer Klub der Jungen 281

Jüdischer Sport- und Schützenverein Haganah 85, 87, 96, 99, 277, 281

Jüdischer Sport- und Schützenverein Menorah; siehe Jüdischer Sport- und Schützenverein Haganah

Jüdischer Sportverband 79-82, 84, 87, 244

Jüdischer Sportverein (JSV) Hasmonea-

Makkabi 81, 82, 85, 87, 281, 282

Jüdischer Turn- und Sportverein Linz 79

Jüdischer Turnverein (JTV) Massada; siehe Makkabi XVII

Jüdischer Turnverein zu Graz; siehe Makkabi-Turnvereine

Kadimah 26, 76, 78, 80-82, 84, 96, 98, 99 , 237

Klagenfurter Athletiksportklub (KAC) 38

Klub der Hakoah-Freunde 296

Kreis Deutschösterreich der jüdischen Turnund Sportvereine $66,70,78,82,84$, $87,88,95,96,276,277,281,282$

Maccabi New York 263

Makkabi-Turnvereine 16, 66, 69-71, 73, 87, $96,98,99,106,277$

- Jüdischer Turnverein Makkabi Graz 73

- Makkabi II 77, 84, 87, 281

- Makkabi IX 73, 84, 87, 88, 281

- Makkabi X 84, 87, 281, 282

- Makkabi XIV 84

- Makkabi XV 73, 87, 88, 281, 284

- Makkabi XVII 77

- Makkabi XIX 84

- Makkabi Groß-Enzersdorf 87

- Makkabi Linz 84

- Makkabi Mödling 87

- Makkabi St. Pölten 87

- Makkabi Wiener Neustadt 87

Makkabi-Weltverband $66,67,70,77,78,87$, $88,95,227,228,276,277,281,282$, 285

Makkabi Wien (1938-1940) 281-285, 287, 296

SC Matzleinsdorf 78

SC Menorah 83 
Naturfreunde 44, 67, 70, 197

FC Nicholson 18

NS-Reichsbund für Leibesübungen (NSRL) 70, 279-281

Olympia 73

SC Orkan 78

Österreichische Bergsteigervereinigung 67, 70

Österreichische Fußball-Union 150

Österreichische Motorrennfahrvereinigung 205

Österreichische Turn- und Sportfront 86, 226, 228-230, 257, 273, 276-279

Österreichischer Amateurbox-Verband 170

Österreichischer Automobil-Club 2

Österreichischer Boxverband 201

Österreichischer Fechtverband 72

Österreichischer Fußball-Bund (ÖFB) 1, 39, 43, 93, 99, 115, 117-119, 121, 123, 150, $171,184,187,190,212,219,248,250$, 252, 256, 258, 259, 263, 280, 299, 306-309, 318, 324, 325

Österreichischer Fußball-Verband; siehe Österreichischer Fußball-Bund

Österreichischer Gebirgsverein 67

Österreichischer Leichtathletikverband 72, 99, 219, 253

Österreichischer Skiverband (ÖSV) 23, 30, 33-35, 207

Österreichischer Skiverein 34, 35

Österreichischer Sportausschuss für leichte Athletik 72

Österreichischer Touring-Club 1

Österreichischer Touristenklub 30, 31, 67

Österreichisches Olympisches Comité (ÖOC) $6,39,72,93,150,220,230,244,252-$ 254,263

Polizeisportvereinigung Wien 294

SK Rapid 21, 40, 107, 114, 117-124, 126, $134,135,141,165,220,241,255,260$, 261, 263, 279

Reichsausschuss jüdischer Sportverbände 284, 285
Salzburger Athletiksportklub (SAK) 38

Schalke 04311

Schweizerischer Fußballverband 256

Schwimmclub Austria 36

SK Slovan 43, 132

Sokolgau 67, 69

Sozialistische Arbeiter-Sportinternationale (SASI) 191, 223, 257, 258

Sparta Prag 310, 311

Sp. C. Herkules (Graz) 203, 204

Sportbund Schild des Reichsbundes jüdischer Frontsoldaten 284, 285

Sportclub Fair 83

Sportklub der Wiener Sportberichterstatter 244

Sportklub jüdischer Studenten 74

Sportklub Wien 1908236

Steirischer Fußballverband 38

SK Sturm Graz 38

Tiroler Fußballverband 38

Tischgesellschaft Wodanbund 41

Tschechoslowakischer Fußballverband 259

Tschechoslowakischer Schwimmverband 228

Turnverein jüdischer Hochschüler; siehe Erster Wiener Jüdischer Turnverein

Turnverein Zion; siehe Makkabi-Turnvereine

Uypest 307

Verband der Amateurfußballvereine Österreichs (VAFÖ) 109, 118, 318

Verband der Arbeiter- und Soldatensportvereinigungen (VAS) $1,79,128,184$, 185, 188, 212, 325, 326

Verband der Sokol-Turnvereine 69

Verband Österreichischer Schwimmvereine (VÖS) 37, 72, 99

Victor Schmidt \& Söhne 93, 244

Vienna Cricket and Football Club 93, 95, 98-101, 104, 105, 118, 125, 142, 164, 170, 235, 238, 239, 243, 305, 306

Vorwärts Hernals 41

SC Wacker 18, 93, 126, 141, 171, 212, 252 Westham United Football Club 252, 330 
Wiener Allround Sportklub 278, 281, 282, 297

Wiener Amateur-Sportverein; siehe FK Austria

Wiener Arbeiter-Turnverein 67, 69

Wiener Association-Football-Club (WAF) 39, 126, 211, 212, 221, 307

Wiener Athletiksport Club (WAC) 1, 36, 71, 72, 83, 96, 99, 101, 104, 105, 126, 152, 155, 164, 237, 322

Wiener Automobil-Club 2

Wiener Cyclisten-Club; siehe Wiener SportClub

Wiener Eislauf-Verein (WEV) 321

Wiener Fußballclub 189873
Wiener Fußball-Verband (WFV) 69, 94, 99, 109, 111, 189, 202, 220, 250, 252, 259, 260, 278, 299, 318

Wiener Golf-Klub 281, 282

Wiener Sport-Club 21, 23, 37, 40-45, 94, 141, 143, 163, 206-208, 210, 211, 253, 317

Wiener Sportvereinigung 41

Young Jewish Sporting Club 77, 80

Zentralstelle der Arbeiter-Turnvereine Österreichs 191

Zentralverband für gemeinsame Sportinteressen 72 